\title{
Mechanisms of Fano Resonances in Coupled Plasmonic Systems
}

\author{
Andrea Lovera, ${ }^{\dagger}$ Benjamin Gallinet, ${ }^{\dagger}$ Peter Nordlander, ${ }^{\ddagger}$ and Olivier J.F. Martin ${ }^{\dagger, *}$ \\ ${ }^{\dagger}$ Nanophotonics and Metrology Laboratory, Swiss Federal Institute of Technology (EPFL), CH-1015 Lausanne, Switzerland and ${ }^{\ddagger}$ Department of Physics and \\ Astronomy, Rice University, MS 61, Houston, Texas 77005, United States
}

\begin{abstract}
Fano resonances in hybridized systems formed from the interaction of bright modes only are reported. Despite precedent works, we demonstrate theoretically and experimentally that Fano resonances can be obtained by destructive interference between two bright dipolar modes out of phase. A simple oscillator model is provided to predict and fit the far-field scattering. The predictions are verified with numerical calculations using a surface integral equation method for a wide range of geometrical parameters. The validity of the model is then further demonstrated with experi-
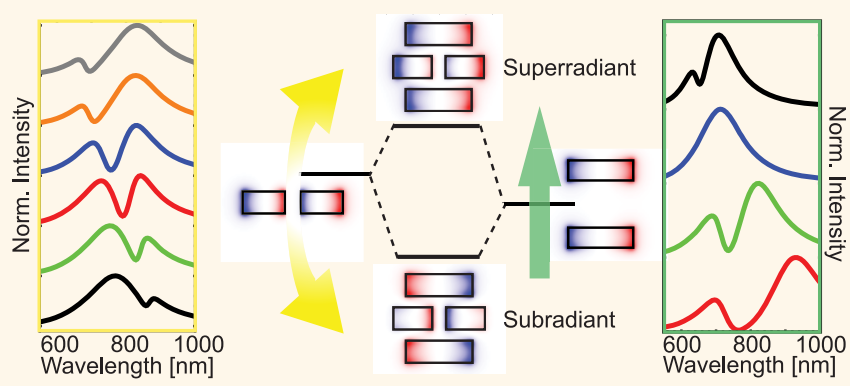

mental dark-field scattering measurements on actual nanostructures in the visible range. A remarkable set of properties like crossings, avoided crossings, inversion of subradiant and superradiant modes and a plasmonic equivalent of a bound state in the continuum are presented. The nanostructure, that takes advantage of the combination of Fano resonance and nanogap effects, also shows high tunability and strong near-field enhancement. Our study provides a general understanding of Fano resonances as well as a simple tool for engineering their spectral features.
\end{abstract}

KEYWORDS: plasmonics · Fano resonance $\cdot$ dipole nanoantenna $\cdot$ radiation damping $\cdot$ oscillator model $\cdot$ hybridization . bound state in the continuum

T he ability of metallic nanostructures to confine and enhance incident radiation offers unique possibilities for manipulating light at the nanoscale. These functionalities are enabled by the excitation of collective electron oscillations known as localized plasmon resonances. ${ }^{1}$ When two or more nanostructures are placed next to each other, their plasmons can couple through near-field interactions and can give rise to a new set of hybridized collective plasmonic modes. ${ }^{2-12}$ Plasmonic nanoclusters composed of three, ${ }^{13}$ four, ${ }^{8,14,15}$ seven particles, ${ }^{9}$ and even larger aggregates ${ }^{11,16}$ can also exhibit interference effects like Fano resonances ${ }^{17-19}$ when the near-field coupling between each element is properly controlled. Fano resonances arise from the interference between superradiant and subradiant modes and produce extinction features with characteristic narrow and asymmetric line shapes. Because of their narrower spectral width compared to standard plasmon resonances and large induced field enhancements, Fano resonances have been used for a variety of applications including plasmonic rulers ${ }^{20-22}$ and biosensors. ${ }^{23-25}$
Despite a large and recent research effort, the design of plasmonic structures exhibiting Fano resonances at specific wavelengths is a challenging task because of their complex nature. A central issue in this design is the spectral engineering of the resonances via controlled hybridization of the available modes. However, this is difficult in systems where higher order modes are excited in the spectral range of interest ${ }^{12,26,27}$ or when the modes are very complex and spatially extend over a large part of the nanostructure. ${ }^{28,29} \mathrm{~A}$ small variation of the geometries, like what can occur during the nanofabrication process, can drastically change the resonance line shape and wavelength. This difficulty is particularly challenging when designing Fano resonant structures using spherical or disk shaped nanoparticles where the energies of the individual nanoparticle plasmons are similar and all hybridization and tuning must be accomplished by controlling the interparticle spacings.

A more robust approach for Fano resonant systems is to engineer them from metallic nanorods that support highly tunable and polarization sensitive longitudinal *Address correspondence to
olivier.martin@epfl.ch.

Received for review March 7, 2013 and accepted April 18, 2013.

Published online April 18, 2013 10.1021/nn401175j

(C) 2013 American Chemical Society 
plasmon modes. ${ }^{30}$ Such nanorods are relatively easy to fabricate in a repeatable and reliable manner and less sensitive to fabrication tolerances. ${ }^{31}$ Furthermore, the controlled interaction between nanorods forming dipole nanoantennas ${ }^{32,33}$ produces a very high field enhancement in the gap, making these structures well suited as ultrasensitive biosensors, ${ }^{34,35}$ plasmonic traps ${ }^{36-38}$ and substrates for surface-enhanced Raman spectroscopy (SERS). ${ }^{39}$ The assembly of nanorods into more complex plasmonic oligomer structures further improves these functionalities and has been pursued by several research groups who have demonstrated Fano interference between electric and magnetic multipolar modes. ${ }^{40-44}$

A second important difficulty in the design of tunable Fano resonant systems is the lack of a simple but comprehensive model. Several recent attempts have been made to address this, leading to very accurate models derived from physical considerations but requiring complex mathematical derivations. ${ }^{45-49}$ Other models, like coupled mechanical oscillators, have been shown to describe Fano interference well, but with the limitation that the damping of the sub- and superradiant modes must be prescribed $a$ priori instead of being calculated explicitly from the radiative characteristics of the individual unhybridized modes. ${ }^{48,50-53}$ While this empirical approach provides significant insight into the nature of the Fano interference, it does not have the predictive power required for use as a design tool.

In this article, we introduce a novel highly tunable Fano resonant nanostructure consisting of four interacting nanorods. In contrast to the "standard" oligomer consisting of nanodisks or nanospheres, the Fano resonance in this structure is caused by interference of the highly tunable dipolar modes of the individual nanorods with no significant involvement of higher multipolar modes. Since the dipolar modes of the nanorods can be tuned by simply changing their aspect ratio, the structure exhibit a remarkable and robust tunability. We also introduce a new extended coupled oscillator model (ECO) for describing Fano interference where the radiative characteristics of the hybridized modes are calculated rather than prescribed a priori. In contrast to the conventional coupled oscillator (CCO) model, ${ }^{54}$ the input parameters in the ECO are the plasmonic properties of the original modes which interact and naturally result in hybridized sub- and superradiant modes with narrow and broad linewidths. We experimentally verify the tunability of the Fano resonance in this structure and demonstrate the applicability of the ECO as an accurate model. First, we show how the geometry of the structure determines the Fano resonance and then how the sign of the coupling between the initial modes reverses the relative energies of the subradiant and superradiant modes, so that the Fano resonance can occur either on the red or blue side of the superradiant mode. This control, which is associated with the near-field properties of the nanostructure, ${ }^{55}$ is very important for practical applications since it provides high design flexibility. Our approach provides new insights into the spectral features of the Fano resonance line shape. In particular, we show that the different spectral features associated with a Fano resonance each correspond to distinct plasmonic modes. Quite remarkably we show that the spectral features on the immediate red and blue sides of a Fano resonance correspond to excitations of the unhybridized modes while the actual Fano resonance corresponds to excitation of the subradiant hybridized mode. This discovery provides crucial insights into how to design Fano resonant structures for use as efficient LSPR sensors and substrates for surface enhanced spectroscopies. Finally, thanks to the tunability of the plasmonic system, we show a plasmonic equivalent of a bound state in the continuum. ${ }^{56,57}$ This effect, which can only be obtained with close interacting modes, is a further proof of the extremely fine control achieved with this system.

\section{RESULTS AND DISCUSSION}

The design we propose is shown in Figure $1 \mathrm{a}$ and is made of a gold dipole nanoantenna (yellow) with a fixed arm length of $70 \mathrm{~nm}$ and a variable gap width surrounded by two gold nanorods (blue). The width of all the components as well as their thickness are fixed to $40 \mathrm{~nm}$ and the lateral distance between antenna and rods has a constant value of $20 \mathrm{~nm}$. The length $L$ of the nanorods is varied from 50 to $150 \mathrm{~nm}$, always keeping the central axes aligned with that of the antenna. A surface integral equation (SIE) method $^{58,59}$ is used for the exact numerical modeling of the optical properties of the different structures. For gold, a realistic dielectric function was taken (Johnson and Christy) ${ }^{60}$ and the surrounding refractive index was set to 1.33 to approximately model the dielectric screening effects of a substrate. This value approximates well the small substrateinduced frequency shift for nanoparticle plasmon resonances polarized parallel to a substrate. ${ }^{33}$ The illumination is taken to be normal to the nanostructure plane, with polarization along the antenna axis.

To understand the origin of the Fano resonance in this system, we first consider the antenna and the nanorods as uncoupled objects supporting independent dipolar resonances. In plasmon hybridization theory, these uncoupled modes are referred to the primitive plasmon modes but will here be referred to the diabatic modes, ${ }^{61}$ i.e., modes that can interact and exhibit a crossing-like characteristic: DA for the antenna and DN for the nanorods. Individually both modes appear bright in the far-field scattering spectra with Lerentzian line shapes. These two modes are graphically shown in Figure S1. DA is the standard antenna mode with the dipoles of the two arms oriented in the same direction. DN is formed by the sum of the dipoles supported by the two external nanorods that oscillate in-phase. Since the nanorods have always the same 

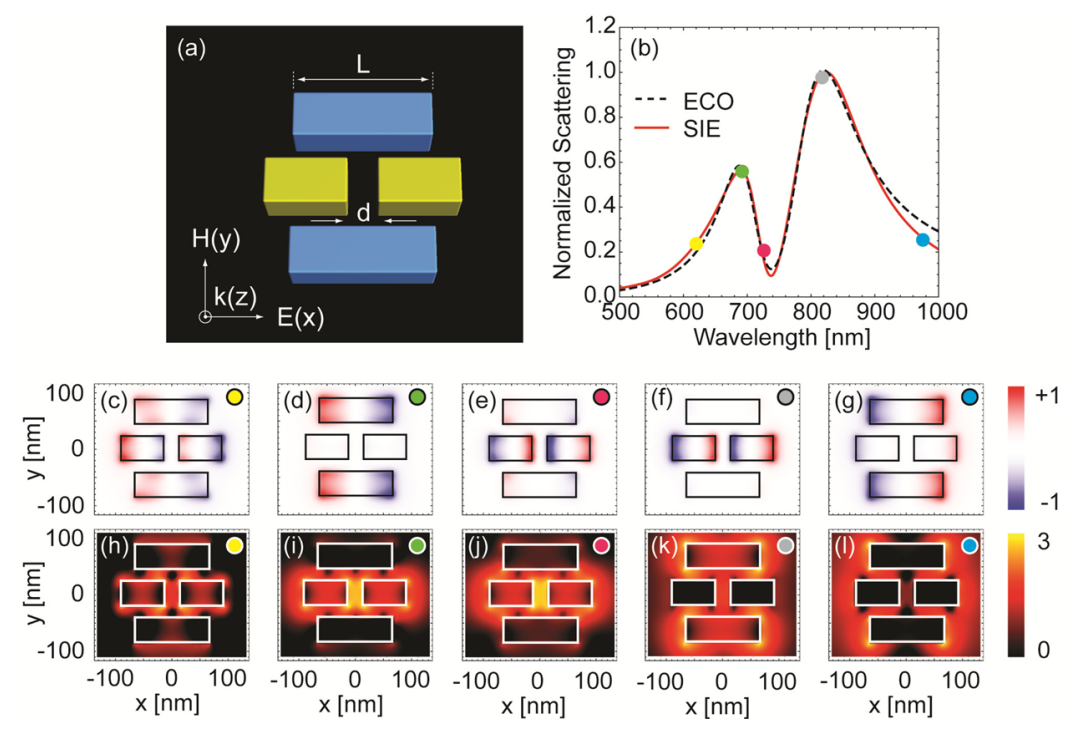

Figure 1. (a) Schematic of the plasmonic structure; the different colors highlight the fact that the system is composed of two independent resonators, one being the dipole antenna (yellow) and the other composed by the two nanorods (blue). The entire structure is made out of gold. (b) SIE simulation (red solid line) and ECO model fit (black dashed line) of the scattering spectrum for a structure with a gap $d=25 \mathrm{~nm}$ and nanorods length $L=120 \mathrm{~nm}$. The parameters extracted from the fit are listed in Table 2. (c-g) Real part of the normal component of the electric field calculated using SIE $5 \mathrm{~nm}$ above the structure at the selected wavelengths shown in panel $b$. The sign of the field gives the charge distribution for the hybridized plasmon modes supported by the structure and visible on the scattering cross section. (h-l) Near-field intensity in logarithmic scale plotted on a plane cutting the structure for the cases shown in panels $\mathbf{c}-\mathrm{g}$.

length, this is the only accessible mode with a plane wave illumination and we can therefore consider the twin nanorods as a single resonator. When the nanostructured elements are combined, with a separation smaller than the illumination wavelength, their plasmon modes interact and form two new hybridized modes. In the terms of molecular orbital theory, these hybridized modes are referred to as adiabatic modes: a superradiant mode (adiabatic bright, $A B$ ) and a subradiant mode (adiabatic dark, $A D$ ). The $A B$ exhibits inphase oscillations of both dipolar modes (antenna and nanorods), resulting in a large total dipole moment which makes the mode highly radiative. On the contrary, the AD has only a small total dipole moment due to the out-of-phase alignment of the dipoles and is a subradiant mode. To better understand the origin of such modes, we remind here that a dipolar resonance is characterized by a phase that matches the phase of the incident field on one side of the resonance and is out-of-phase on the other side. In the coupled system under study, when DA and DN have different resonances, there are some regions of the spectrum where their phases are matched, forming a stronger resonant mode $(A B)$ with higher radiance because of constructive interference. On the other hand, in the spectral region where they are out-of-phase, because of destructive interference, a low radiant or subradiant mode is formed (AD, see Supporting Information Figure S2). The Fano resonance results from the interference of these adiabatic modes that overlap both spectrally and spatially. In Figure 1b, we show the scattering spectrum for a structure with nanorods length $L=120 \mathrm{~nm}$ and antenna gap $d=25 \mathrm{~nm}$ that reveal a typical Fano resonance line shape.

The validity of the hybridization model is supported by the charge distribution analysis of the plasmon modes that appear at specific frequencies. A simple way of visualizing the charges is to compute the normal component of the electric field in a plane $5 \mathrm{~nm}$ above the structure, since its orientation is directly related to the sign of the charges. From Figure 1e we see that the related mode profile is the one expected from the hybridization diagram: antenna and nanorods support electron oscillations that are dipolar and outof-phase, producing a very small total dipole moment of subradiant nature. In this case, we observe as well a maximum in the near-field intensity located in the antenna gap, Figure $1 \mathrm{j}$, that can be up to $40 \%$ stronger than the field computed at the same point for an isolated antenna at the resonance (see Supporting Information Figure S3). The AD mode, whose position is determined by a maximum in the absorption spectrum (computed using the ohmic losses method, ${ }^{62}$ Supporting Information Figure S4), is then mainly supported by the antenna with a weak contribution of the side nanorods. Away from the Fano resonance, at the wavelengths corresponding to (c) and (g), the charge distribution is associated with the tails of the superradiant mode characterized by in-phase dipolar excitation of the plasmonic structure constituents. As expected from classical dipolar resonances, there is a $\pi$ phase difference between the charge distributions at (c) and (g). The related near-field shows that the major contribution to the mode is provided by the nanorods 
at long wavelength (I), where a field intensity maximum is recorded at their extremities. The peak of $A B$, determined from the absorption spectrum, is at $\lambda=$ $758.8 \mathrm{~nm}$. Most interestingly, the charge distribution analysis reveals that at the peaks surrounding the Fano resonance, the charge distributions correspond to the diabatic modes, DA (d) and DN (f) with phases matching the external illumination. The related near-field analysis further highlights this conclusion, showing that the intensity enhancement is located mainly around the antenna (i) and nanorods (k), respectively. This shows that, even if the system is strongly hybridized, the diabatic modes can be excited with appropriate wavelengths. This finding, which has been recently also shown for a different system, ${ }^{63}$ is extremely important, since it enables a detailed spatial tuning of the near-field with frequency. We believe that the finding that the diabatic modes can be excited directly by driving the system at the frequencies of the two spectral peaks surrounding the Fano resonances is a general feature of plasmonic systems.

To provide better insights into the system, we now develop an extended coupled oscillator (ECO) model for the plasmonic response of coupled plasmonic structures, Figure 2a. The conventional coupled oscillator (CCO) model consists of two damped oscillators coupled with a spring of constant g. The two modes refer to the hybridized broad superradiant and the narrow subradiant mode. Fano interference results in the scattering spectrum by driving only the superradiant mode with an external field. The damping of the two oscillators is prescribed a priori. In contrast to the CCO, the oscillators in our ECO model here represent the original diabatic modes of the system and are characterized by resonant frequencies $\omega_{1}, \omega_{2}$ and damping $\gamma_{1}, \gamma_{2}$ accounting for nonradiative losses. Nonradiative damping in a nanoparticle is a local quantity that only depends on the local plasmon oscillation and is independent of any dissipation in nearby systems. In contrast, radiative damping is a collective effect exhibiting interference, i.e.. sub- or superradiance. The radiative damping of two dipolar oscillators is proportional to the total dynamical dipole moment of the system. To describe the radiative damping, we express the total dipole moment of the system as $P_{\text {tot }}=P_{1}+P_{2}=\alpha_{1} x_{1}+\alpha_{2} x_{2}$, where $P_{1,2}$ are the dipole moments of oscillators 1 and $2, x_{1,2}$ are their respective amplitudes and $\alpha_{1,2}$ are polarizabilities relating the diabatic plasmon amplitudes to their induced dipole moments. The magnitude of each dipole moment can be positive or negative, depending on how the amplitudes $x_{1,2}$ are defined relative to the external field. The external excitation is represented by forces applied on both oscillators and is here for simplicity assumed to be in phase, which can be justified when the spatial extension of the system is smaller than a quarter wavelength of the incident light. The net force on each oscillator is proportional to their polarizabilities $F_{1,2}=\alpha_{1,2} E_{\text {ext }}$.

The equations of motion can then be written as follows:

$$
\left\{\begin{array}{l}
\ddot{x}_{1}+\gamma_{1} \dot{x}_{1}+\omega_{1}^{2} x_{1}+g x_{2}=0.5 \dddot{P}_{\text {tot }}+\alpha_{1} E_{\text {ext }} \\
\ddot{x}_{2}+\gamma_{2} \dot{x}_{2}+\omega_{2}^{2} x_{2}+g x_{1}=0.5 \dddot{P}_{\text {tot }}+\alpha_{2} E_{\text {ext }}
\end{array}\right.
$$

The radiative term $P_{\text {tot }}$ introduces a coupling between each oscillator. If the motion of the oscillator is out-ofphase, radiative damping is suppressed, while for inphase motion, radiative damping is enhanced. This effect is the classical analogue of sub- and superradiance. This model has general validity, and in specific limiting cases, we recover already established models. If $\alpha_{2} \ll \alpha_{1}$, we recover the CCO: ${ }^{48,50,52}$ oscillator 1 is fully forced and has both radiative and nonradiative contributions to the losses; oscillator 2 has no radiative losses and is not forced. If $\alpha_{2} \ll \alpha_{1}$ and $g=0$, we recover the model proposed by Kats et al. ${ }^{64}$ that describes the behavior of a single oscillator with radiative damping.

For an incident harmonic field, $E_{\text {ext }}=E_{0} \mathrm{e}^{\mathrm{i} \omega \mathrm{t}}$, the displacements $x_{1}$ and $x_{2}$ of the oscillators are harmonic with $x_{1,2}(\omega)=C_{1,2}(\omega) \mathrm{e}^{\mathrm{i} \omega \mathrm{t}}$, where $C_{1,2}$ are the oscillation amplitudes. $C_{1,2}$ can be computed analytically from eq 1 and provide information on the frequency response of the system. The squared modulus of the sum of the amplitudes, $\left|C_{1}+C_{2}\right|^{2}$, represents light scattering efficiency of the system and is used to model the scattering spectrum. A fit of the scattering spectrum for the system in Figure 1 is shown with the black dashed line in Figure $1 b$, demonstrating an excellent agreement with the SIE results.

With this ECO, we can also reconstruct the charge polarization of the system presented in Figure $1 \mathrm{c}-\mathrm{g}$. First, we compute a near-field map of the $z$-component of the electric field for a single frequency, far away from the resonance, for each of the uncoupled antenna alone and the nanorods alone (Supporting Information Figure S1). We then multiply each map, after normalization, by the corresponding oscillator amplitudes $C_{1,2}(\omega)$ extracted from the oscillator model. Figure $2 \mathrm{~b}-\mathrm{f}$ shows the calculated charge distributions, which are in almost perfect agreement with the results in Figure 1c-g obtained from full electromagnetic calculations. Also here we see that, by changing the frequency of the applied field, we can selectively excite both the diabatic and adiabatic modes of the system. Since $C_{1,2}$ are complex numbers, they do not only provide amplitude but also phase information. When their real parts have the same sign, the two oscillators resonate in-phase, while out-of-phase oscillations are produced for opposite signs (Supporting Information Figure S5).

The geometry of the plasmonic structure plays a major role in determining the energies of the diabatic and adiabatic modes. In the following, we explore the 

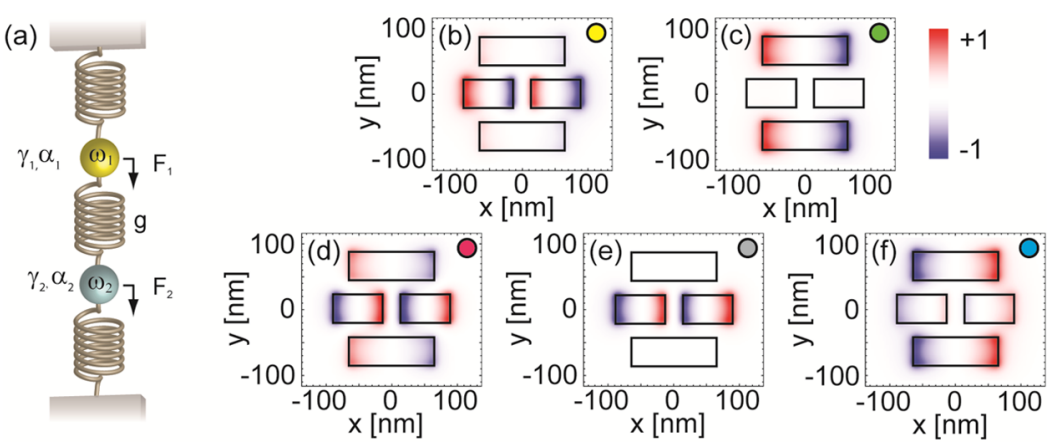

Figure 2. (a) Schematics of the ECO model proposed for the fitting. The oscillators correspond to the diabatic modes DA and DN. $(b-f)$ Reconstruction of the near-field map using ECO model. This shows excellent agreement compared with the maps obtained with the SIE method and presented in Figure 1c-g. Each plot is obtained by sampling the electric field at a single wavelength far from the resonance on a plane $5 \mathrm{~nm}$ above the structures for isolated antenna and nanorods multiplied by $C_{1,2}$ extracted from the model.

tunability of the Fano resonance by varying the geometry. Figure 3 shows the effect of tuning the antenna gap on the scattering spectrum of the system. The high resolution scanning electron microscope (SEM-SFEG Sirion) pictures show examples of geometries with gap sizes between 5 and $50 \mathrm{~nm}$. The structures are fabricated with e-beam lithography followed by $2 \mathrm{~nm} \mathrm{Ti}$ (adhesion layer) and $40 \mathrm{~nm}$ Au thermal evaporation on a glass substrate and lift-off process.

The experimental dark-field scattering measurements on single structures are shown in Figure 3a which agree very well with the SIE calculations in Figure $3 \mathrm{~b}$ and the ECO results in Figure $3 \mathrm{c}$. The experimental spectra are a little broader and damped than the simulated spectra which is most likely caused by defects generated by the lithographic fabrication process. The analysis of the scattering spectra reveals two main features. The first one is a blue shift of the peak associated with DA, which can be explained by the fact that the gap influences the resonance position of the antenna. ${ }^{33}$ The second and more important effect is the spectral inversion of the superradiant and the subradiant modes as the gap size is reduced. For large gaps, the Fano resonance (AD) appears on the blue side of the main scattering resonance $(A B)$, but for small gap sizes, the positions are reversed. This indicates a direct relation between the gap width and the coupling between the two resonators. In fact, in a coupled system, the relative positions of the hybridized modes depend on the sign and strength of the coupling. $^{61}$

The plasmon hybridization graph in Figure 3e provides a synthetic view of the results in Figure $3 \mathrm{~b}$. The figure clearly shows that the hybridized modes exhibit an anticrossing and an energy inversion for a gap width in between 20 and $22.5 \mathrm{~nm}$. The relative energies of the hybridized modes are again extracted from the absorption cross section of the coupled systems. The inversion is also confirmed by the flipping of the absorption peaks corresponding to the two modes (Supporting
Information Figure 56 ). It should be noticed that the $A D$ mode (blue line) lies above the DA (gray dashed line) which is contrary to what would be expected in electrostatic hybridization theory. The reason for this is retardation effects which induce a redshift (dynamical depolarization) of the collective hybridized mode. Such a redshift of a hybridized antibonding mode with respect to the parent unhybridized mode (DA) also occurs for nanoshells. $^{2}$

In Table 1, we report the fitting parameters for the ECO model. These data together with Figure $3 f$ clearly show a dependence of the coupling coefficient $g$ on the gap width. Note also the change of sign in the coupling coefficient, leading to a reversal of the Fano resonance energy with respect to the superradiant mode. For zero coupling, the adiabatic modes are not defined and there is a discontinuous jump in Figure 3e. This is a fundamentally different behavior from standard avoided crossings, where the interaction remains finite and the two adiabatic states repel each other. The unusual phenomenon of a sign change in the coupling between the antenna and nanorod modes can be understood from the schematic in Figure 3g: as the antenna gap increases, the Coulomb interaction changes sign from repulsive to attractive.

Let us now consider the influence of the nanorods length on the response of the system. Normalized experimental scattering spectra as well as SIE simulations with corresponding oscillator model fitting and SEM pictures for four different nanorods lengths are presented in Figure 4. The calculated spectra agree very well with the measured spectra as well as with the ECO model. Several effects caused by the tuning can be immediately observed: a clear red shift of the superradiant peak, a width tuning of the Fano resonance and the disappearance of the Fano resonance for a specific nanorods length. The first effect is simply due to the fact that increasing the nanorods length causes a red shift of the DN mode and a resulting red shift of the hybridized modes. Since the width of the dip is determined by the energy difference between the two diabatic states, red-shifting DN with respect to DA 


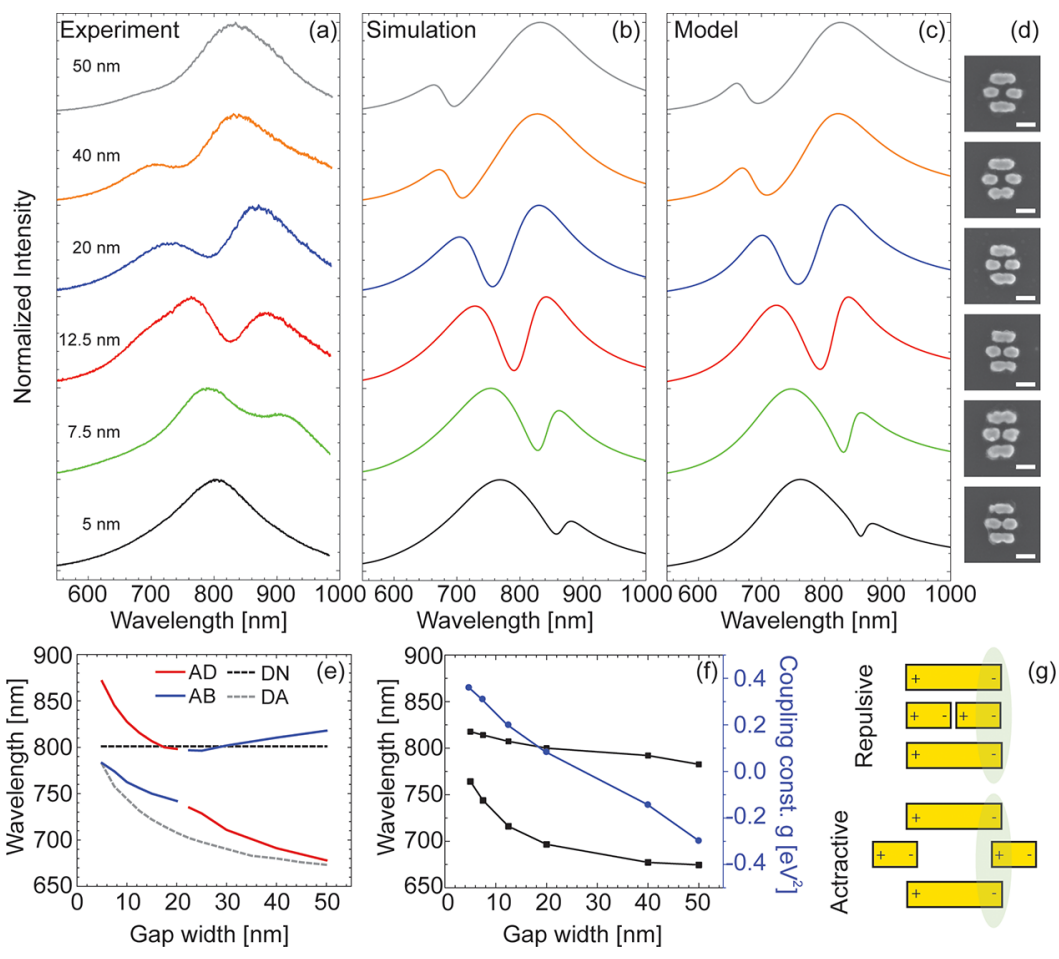

Figure 3. (a) Dark-field measurements, (b) SIE scattering simulations, (c) fit with the ECO model, and (d) SEM pictures (scale bar $100 \mathrm{~nm}$ ) of structures with constant nanorods length $L=120 \mathrm{~nm}$ and increasing antenna gap size from 5 to $50 \mathrm{~nm}$. (e) Plasmon hybridization diagram with the spectral position of the diabatic (dashed lines) and adiabatic modes (solid red and blue lines) versus the gap width. (f) Resonance wavelengths of the diabatic modes extracted from the fitting with ECO (black) and coupling constant $g$ (blue). (g) Schematics of Coulomb interaction for different gap widths. For small gaps, the AB mode is at higher energy than AD because of repulsive forces between antenna and nanorods, while for large gaps, we have attractive forces and the opposite effect.

TABLE 1. Extracted Parameters from ECO Model for Varying Gap Widths (Figure 3)

gap width $\omega_{1}[\mathrm{eV}] \omega_{2}[\mathrm{eV}] \quad \boldsymbol{g}\left[\mathrm{eV}^{2}\right] \quad E_{0} \quad \gamma_{1}[\mathrm{eV}] \gamma_{2}[\mathrm{eV}] \alpha_{1}\left[\mathrm{eV}^{-1}\right] \alpha_{2}\left[\mathrm{eV}^{-1}\right]$

$\begin{array}{lllllllll}5 \mathrm{~nm} & 1.516 & 1.623 & 0.361 & 2.863 & 0.096 & 0.074 & 0.110 & 0.140\end{array}$

$\begin{array}{lllllllll}7.5 \mathrm{~nm} & 1.523 & 1.667 & 0.311 & 2.891 & 0.050 & 0.066 & 0.110 & 0.140\end{array}$

$\begin{array}{lllllllll}12.5 \mathrm{~nm} & 1.536 & 1.732 & 0.200 & 3.025 & 0.064 & 0.064 & 0.109 & 0.139\end{array}$

$\begin{array}{lllllllll}20 \mathrm{~nm} & 1.550 & 1.780 & 0.084 & 2.773 & 0.083 & 0.051 & 0.128 & 0.131\end{array}$

$\begin{array}{lllllllll}40 \mathrm{~nm} & 1.566 & 1.831 & -0.143 & 3.626 & 0.155 & 0.070 & 0.101 & 0.064\end{array}$

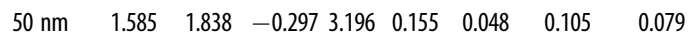

increases the width of the Fano dip and allows a precise control of the transparency window.

In Figure $4 \mathrm{e}$, we present a plasmon hybridization diagram that clearly illustrates the effects of tuning the energy of DN. As the uncoupled nanorod resonance (DN, black dashed line) wavelength approaches that of the uncoupled antenna (DA, gray dashed line), the hybridized modes exhibit a clear avoided crossing behavior. Again, the positions of $A B$ and $A D$ are extracted from the absorption peaks. For the same reason as in Figure $3 e$, the hybridized modes do not converge asymptotically to DA and DN for long nanorods. The anticrossing behavior implies that the magnitude and sign of the coupling remains unchanged. This is confirmed by the values extracted from ECO fitting in Table 2.
Surprisingly, when the diabatic modes cross, which occurs for $L \sim 87.5 \mathrm{~nm}$, the width of the Fano resonance goes to a minimum and the Fano line shape is completely damped, Figure 4, leading to a single Lorentzian peak for the scattering cross section that corresponds then to a pure bright mode. This effect, which is exclusively associated with the positions of DA and DN and not with any other parameter like the coupling, is the plasmonic equivalent of a quantum mechanical effect known in literature as bound state in the continuum (BIC). ${ }^{56,57}$ This system is in fact equivalent to two states that are in interaction with radiation. The tunability of plasmonic systems enables positioning the diabatic states very close to each other while maintaining appreciable coupling. Under these conditions, a narrow bound state with infinite radiative lifetime but a total finite lifetime because of Ohmic losses is formed in the continuum. Figure $4 \mathrm{f}$ shows this effect clearly: the width associated with the AD mode goes toward the minimum for nanorod lengths approaching $87.5 \mathrm{~nm}$ while the width of the $A B$ mode reaches a maximum. Because of the overlap of the absorption peaks around the anticrossing point, the modal widths cannot be extracted from the absorption spectra but are instead determined using the Gallinet formula ${ }^{65}$ that provide position and mode width of the hybridized modes by fitting the scattering cross 

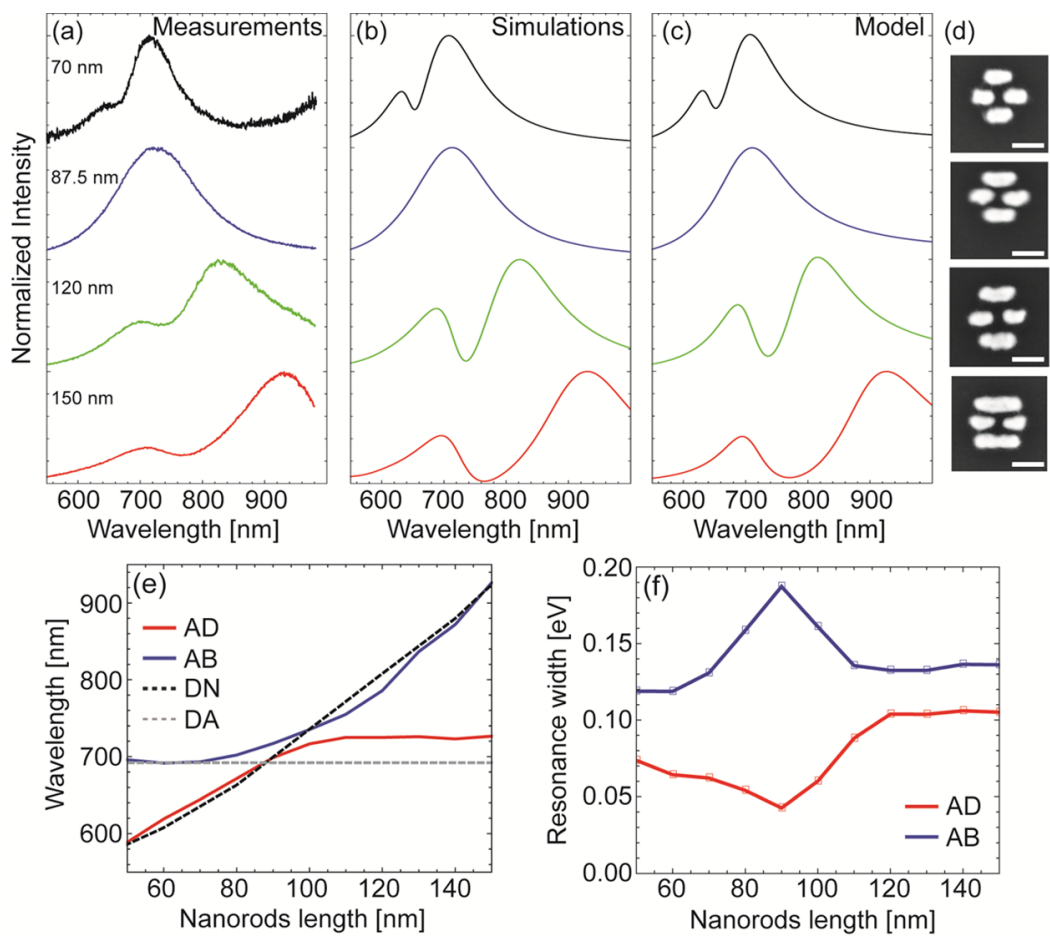

Figure 4. (a) Dark-field measurements, (b) SIE simulations, (c) fit with ECO model, and (d) SEM pictures (scale bar $100 \mathrm{~nm}$ ) of systems with different nanorods lengths ranging from 70 to $150 \mathrm{~nm}$ and antenna gap fixed at $25 \mathrm{~nm}$. (e) Plasmon hybridization diagram with the spectral position of diabatic and adiabatic modes versus nanorods length. (f) $A B$ and $A D$ mode resonance widths versus nanorods length extracted with a fitting using Gallinet formula.

TABLE 2. Parameters for the ECO Model for Varying Nanorods Lengths (Figure 4)

\begin{tabular}{lcccccccc}
$\begin{array}{l}\text { nanorods } \\
\text { length }\end{array}$ & $\omega_{\mathbf{1}}[\mathrm{eV}]$ & $\omega_{\mathbf{2}}[\mathrm{eV}]$ & $\boldsymbol{g}\left[\mathrm{eV}^{2}\right]$ & $\boldsymbol{E}_{\mathbf{0}}$ & $\gamma_{\mathbf{1}}[\mathrm{eV}]$ & $\gamma_{\mathbf{2}}[\mathrm{eV}]$ & $\alpha_{\mathbf{1}}\left[\mathrm{eV}^{-\mathbf{1}}\right]$ & $\alpha_{\mathbf{2}}\left[\mathrm{eV}^{-\mathbf{1}}\right]$ \\
\hline $70 \mathrm{~nm}$ & 1.938 & 1.799 & -0.140 & 4.607 & 0.081 & 0.111 & 0.065 & 0.067 \\
$87.5 \mathrm{~nm}$ & 1.799 & 1.799 & -0.020 & 3.122 & 0.045 & 0.045 & 0.110 & 0.110 \\
$120 \mathrm{~nm}$ & 1.554 & 1.798 & -0.025 & 3.070 & 0.100 & 0.068 & 0.100 & 0.112 \\
$150 \mathrm{~nm}$ & 1.380 & 1.767 & -0.130 & 2.830 & 0.148 & 0.088 & 0.095 & 0.115
\end{tabular}

section. A near-field analysis of the structure also reveals a minimum in the electric field enhancement of the $A D$ compared to the field enhancement of structures with different nanorod lengths (Supporting Information Figure S3) further highlighting the disappearance of the AD mode. This behavior can also be well reproduced with the oscillator model. In this case, we must impose the same resonance frequency for both oscillators $\left(\omega_{1}=\omega_{2}\right)$. For simplicity, we assume that $\gamma_{1}=\gamma_{2}$ and $\alpha_{1}=\alpha_{2}$. For an in-phase excitation, we can solve eq 1 and find the total oscillation amplitude:

$$
\left|C_{1}+C_{2}\right|^{2}=\left|\frac{2 E_{0} \alpha_{1}}{g-\omega^{2}+\omega_{1}^{2}+i \omega\left(\omega^{2} \alpha_{1}+\gamma_{1}\right)}\right|^{2}
$$

Equation 2 describes a single resonance with Lorentzian line shape centered at $\omega_{b}^{2}=\omega_{1}^{2}+g$ with a line width $\Gamma_{b}=\alpha_{1} \omega^{2}+\gamma_{1}$. The fact that the width contains both the radiative and nonradiative coefficients confirms the hypothesis that we are exciting the superradiant mode. We can also use the ECO model to probe the subradiant mode, whose amplitude is determined by the difference of $C_{1}$ and $C_{2}$ when imposing $F_{1}=-F_{2}$, corresponding to an out-of-phase excitation. ${ }^{66,67}$ With this excitation condition, the solution of eq 1 becomes

$$
\left|C_{1}-C_{2}\right|^{2}=\left|\frac{2 E_{0} \alpha_{1}}{-g-\omega^{2}+\omega_{1}^{2}+i \omega \gamma_{1}}\right|^{2}
$$

We obtain again a resonance with a Lorentzian profile, but now centered at $\omega_{d}^{2}=\omega_{1}^{2}-g$ and with $\Gamma_{d}=\gamma_{1}$. The resonance width is now determined only by nonradiative losses and is therefore much narrower than the superradiant mode. The radiative losses cancel out, in perfect agreement with experimental work on Fano resonances. ${ }^{68}$ Equation 3, together with Figure $4 f$ also explains the increase of the width of the Fano resonance, from the minimum value given by nonradiative losses, to the maximal asymptotic value given by both contributions $\gamma$ and $\alpha$. Comparing the hybridized mode positions, we see that their respective frequencies are $\omega_{b, d}=\omega_{1} \pm g$. This result that is in line with classical strong coupling theory ${ }^{61}$ is significant and also confirms the anticrossing nature of the hybridized modes.

Finally, as seen in Figures 3 and 4, this system provides a very simple way of tuning the Fano line shape in hybridized systems. Both the position and modulation depth can be precisely controlled by using only two geometrical parameters and a condition for 
the best near-field enhancement can be obtained ${ }^{22}$ also thanks to the combination of antenna nanogap and efficient energy storage of a Fano system (Supporting Information Figure S3).

\section{CONCLUSIONS}

A novel gold plasmonic nanostructure that combines dipolar resonances into a hybrid system supporting a Fano resonance in the visible range of the spectrum has been studied both numerically and experimentally. The nanostructure exhibits high tunability and robust control of its spectral features with only a few structural parameters. The analysis of the charge distribution revealed that the uncoupled modes forming the hybridized modes can be excited for specific frequencies close to the peaks surrounding the Fano resonance in the scattering spectra. This finding implies that the location of the hot spots in the nanostructure is dependent on the illumination wavelength. An extended oscillator model that includes radiation damping has been introduced to fit experimental and numerical data. This simple model predicts the behavior of the plasmonic system extremely well. Experimental data have shown both crossing and avoided crossing behavior as a consequence changing the gap or the length of the nanorods. In particular, this structure allows for an inversion of the relative positions of the subradiant and superradiant modes with the antenna gap modulation. By tuning the length of the nanorods, we have demonstrated a plasmonic equivalent of a bound state in the continuum.

\section{METHODS}

In the experiments, we have used an inverted dark field optical microscope equipped with an oil immersion $60 \times$ objective with a numerical aperture of 1.45 (PLAPON 60xO TIRFM, Olympus). The sample was placed on top of the objective on a piezoelectric stage (ND-MTD, Ntegra). The illumination source was a halogen lamp and a polarizer (LPVIS050-MP, Thorlabs) was used to set the polarization of the beam parallel to the main dimension of the antennas. To maintain the light polarized at the sample plane, the beam position was adjusted so that the reflection on an annular gold mirror was only on one side. The scattered light was collected by a spectrometer (Shamrock SR-303i, Andor) equipped with an electronic cooled CCD camera (iDus 401BRDD, Andor).

Conflict of Interest: The authors declare no competing financial interest.

Acknowledgment. It is a pleasure to acknowledge funding from the CCMX-FanoSense project, the Swiss National Science Foundation (200021-125326) and from the European Community's Seventh Framework Program (FP7-ICT-2009-4, Grant agreement 248835). P.N. acknowledges support from the Robert A. Welch foundation (C-1222) and the Defense Threat Reduction Agency (HDTRA1-11-1-0040).

Supporting Information Available: A schematics explaining the hybrid modes formations and additional figures of absorption cross sections of different geometries, near-field maps in specific points around the nanostructure and on planes on top of isolated antenna and nanorods, reconstruction of a scattering cross section using the ECO model as well as the plot of the real part of $C_{1}$ and $C_{2}$. This material is available free of charge via the Internet at http://pubs.acs.org.

\section{REFERENCES AND NOTES}

1. Maier, S. A. Plasmonics: Fundamentals and Applications; Spinger: New York, 2007.

2. Prodan, E.; Radloff, C.; Halas, N. J.; Nordlander, P. A Hybridization Model for the Plasmon Response of Complex Nanostructures. Science 2003, 302, 419-422.

3. Jain, P. K.; Eustis, S.; El-Sayed, M. A. Plasmon Coupling in Nanorod Assemblies: Optical Absorption, Discrete Dipole Approximation Simulation, and ExcitonCoupling Model. J. Phys. Chem. B 2006, 110, 1824318253.

4. Jain, P. K.; Huang, W.; El-Sayed, M. A. On the Universal Scaling Behavior of the Distance Decay of Plasmon Coupling in Metal Nanoparticle Pairs: A Plasmon Ruler Equation. Nano Lett. 2007, 7, 2080-2088.
5. Olk, P.; Renger, J.; Wenzel, M. T.; Eng, L. M. Distance Dependent Spectral Tuning of Two Coupled Metal Nanoparticles. Nano Lett. 2008, 8, 1174-1178.

6. Jain, P. K.; El-Sayed, M. A. Surface Plasmon Coupling and Its Universal Size Scaling in Metal Nanostructures of Complex Geometry: Elongated Particle Pairs and Nanosphere Trimers. J. Phys. Chem. C 2008, 112, 4954-4960.

7. Funston, A. M.; Novo, C.; Davis, T. J.; Mulvaney, P. Plasmon Coupling of Gold Nanorods at Short Distances and in Different Geometries. Nano Lett. 2009, 9, 1651-1658.

8. Fan, J. A.; Wu, C.; Bao, K.; Bao, J.; Bardhan, R.; Halas, N. J.; Manoharan, V. N.; Nordlander, P.; Shvets, G.; Capasso, F. Self-Assembled Plasmonic Nanoparticle Clusters. Science 2010, 328, 1135-1138.

9. Hentschel, M.; Saliba, M.; Vogelgesang, R.; Giessen, H.; Alivisatos, A. P.; Liu, N. Transition from Isolated to Collective Modes in Plasmonic Oligomers. Nano Lett. 2010, 10, 2721-2726.

10. Davis, T. J.; Gómez, D. E.; Vernon, K. C. Simple Model for the Hybridization of Surface Plasmon Resonances in Metallic Nanoparticles. Nano Lett. 2010, 10, 2618-2625.

11. Hentschel, M.; Dregely, D.; Vogelgesang, R.; Giessen, H.; Liu, N. Plasmonic Oligomers: The Role of Individual Particles in Collective Behavior. ACS Nano 2011, 5, 2042-2050.

12. Woo, K. C.; Shao, L.; Chen, H.; Liang, Y.; Wang, J.; Lin, H.-Q. Universal Scaling and Fano Resonance in the Plasmon Coupling between Gold Nanorods. ACS Nano 2011, 5, 5976-5986.

13. Brandl, D. W.; Mirin, N. A.; Nordlander, P. Plasmon Modes of Nanosphere Trimers and Quadrumers. J. Phys. Chem. B 2006, 106, 12302-12310.

14. Fan, J. A.; Bao, K.; Wu, C.; Bao, J.; Bardhan, R.; Halas, N. J.; Manoharan, V. N.; Shvets, G.; Nordlander, P.; Capasso, F. Fano-Like Interference in Self-Assembled Plasmonic Quadrumer Clusters. Nano Lett. 2010, 10, 4680-4685.

15. Rahmani, M.; Lei, D. Y.; Giannini, V.; Lukiyanchuk, B.; Ranjbar, M.; Liew, T. Y. F.; Hong, M.; Maier, S. A. Subgroup Decomposition of Plasmonic Resonances in Hybrid Oligomers: Modeling the Resonance Lineshape. Nano Lett. 2012, 6, 2101-2106.

16. Dregely, D.; Hentschel, M.; Giessen, H. Excitation and Tuning of Higher-Order Fano Resonances in Plasmonic Oligomer Clusters. ACS Nano 2011, 5, 8202-8211.

17. Verellen, N.; Sonnefraud, Y.; Sobhani, H.; Hao, F.; Moshchalkov, V. V.; Van Dorpe, P.; Nordlander, P.; Maier, S. A. Fano Resonances in Individual Coherent Plasmonic Nanocavities. Nano Lett. 2009, 9, 1663-1667.

18. Miroshnichenko, A. E.; Flach, S.; Kivshar, Y. S. Fano Resonances in Nanoscale Structures. Rev. Mod. Phys. 2010, 82, 2257-2298. 
19. Rahmani, M.; Luk'yanchuk, B.; Hong, M. Fano Resonance in Novel Plasmonic Nanostructures. Laser Photonics Rev. 2012, 10.1002/lpor.201200021.

20. Liu, N.; Hentschel, M.; Weiss, T.; Alivisatos, A. P.; Giessen, H. Three-Dimensional Plasmon Rulers. Science 2011, 332, 1407-1410.

21. Shao, L.; Fang, C.; Chen, H.; Man, Y. C.; Wang, J.; Lin, H.-Q. Distinct Plasmonic Manifestation on Gold Nanorods Induced by the Spatial Perturbation of Small Gold Nanospheres. Nano Lett. 2012, 12, 1424-1430.

22. Gallinet, B.; Siegfried, T.; Sigg, H.; Nordlander, P.; Martin, O. Plasmonic Radiance: Probing Structure at the Angstrom Scale with Visible Light. Nano Lett. 2013, 13, 497-503.

23. Verellen, N.; Van Dorpe, P.; Huang, C.; Lodewijks, K.; Vandenbosch, G. A. E.; Lagae, L.; Moshchalkov, V. V. Plasmon Line Shaping Using Nanocrosses for High Sensitivity Localized Surface Plasmon Resonance Sensing. Nano Lett. 2011, 11, 391-397.

24. Wu, C.; Khanikaev, A. B.; Adato, R.; Arju, N.; Yanik, A. A.; Altug, H.; Shvets, G. Fano-Resonant Asymmetric Metamaterials for Ultrasensitive Spectroscopy and Identification Ofâ molecular Monolayers. Nat. Mater. 2011, 11, 69-75.

25. Ye, J.; Wen, F.; Sobhani, H.; Lassiter, J. B.; Van Dorpe, P.; Nordlander, P.; Halas, N. J. Plasmonic Nanoclusters: Near Field Properties of the Fano Resonance Interrogated with Sers. Nano Lett. 2012, 12, 1660-1667.

26. Brown, L. V.; Sobhani, H.; Lassiter, J. B.; Nordlander, P.; Halas, N. J. Heterodimers: Plasmonic Properties of Mismatched Nanoparticle Pairs. ACS Nano 2010, 4, 819-832.

27. Hao, F.; Nordlander, P.; Sonnefraud, Y.; Van Dorpe, P.; Maier, S. A. Tunability of Subradiant Dipolar and FanoType Plasmon Resonances in Metallic Ring/Disk Cavities: Implications for Nanoscale Optical Sensing. ACS Nano 2009, 3, 643-652.

28. Liu, S.-D.; Yang, Z.; Liu, R.-P.; Li, X.-Y. Multiple Fano Resonances in Plasmonic Heptamer Clusters Composed of Split Nanorings. ACS Nano 2012, 6, 6260-6271.

29. Sonnefraud, Y.; Verellen, N.; Sobhani, H.; Vandenbosch, G. A. E.; Moshchalkov, V. V.; Van Dorpe, P.; Nordlander, P.; Maier, S. A. Experimental Realization of Subradiant, Superradiant, and Fano Resonances in Ring/Disk Plasmonic Nanocavities. ACS Nano 2010, 4, 1664-1670.

30. Fischer, H.; Martin, O. J. F. Polarization Sensitivity of Optical Resonant Dipole Antennas. J. Eur. Opt. Soc. Rapid Publ. 2008, 3, 08018.

31. Kern, A. M.; Martin, O. J. F. Excitation and Reemission of Molecules near Realistic Plasmonic Nanostructures. Nano Lett. 2011, 11, 482-487.

32. Muhlschlegel, P.; Eisler, H.-J.; Martin, O. J. F.; Hecht, B.; Pohl, D. W. Resonant Optical Antennas. Science 2005, 308, 1607-1608.

33. Fischer, H.; Martin, O. J. F. Engineering the Optical Response of Plasmonic Nanoantennas. Opt. Express 2008, 16, 9144-9154.

34. Aćimović, S. S.; Kreuzer, M. P.; González, M. U.; Quidant, R. Plasmon Near-Field Coupling in Metal Dimers as a Step toward Single-Molecule Sensing. ACS Nano 2009, 3, 12311237.

35. Rosi, N. L.; Mirkin, C. A. Nanostructures in Biodiagnostics. Chem. Rev. 2005, 105, 1547-1562.

36. Zhang, W.; Huang, L.; Santschi, C.; Martin, O. J. F. Trapping and Sensing $10 \mathrm{~nm}$ Metal Nanoparticles Using Plasmonic Dipole Antennas. Nano Lett. 2010, 10, 1006-1011.

37. Righini, M.; Ghenuche, P.; Cherukulappurath, S.; Myroshnychenko, V.; Garcia de Abajo, F. J.; Quidant, R. NanoOptical Trapping of Rayleigh Particles and Escherichia coli Bacteria with Resonant Optical Antennas. Nano Lett. 2009, 9, 3387-3391.

38. Grigorenko, A. N.; Roberts, N. W.; Dickinson, M. R.; Zhang, Y. Nanometric Optical Tweezers Based on Nanostructured Substrates. Nat. Photonics 2008, 2, 365-370.

39. Zhang, W. H.; Fischer, H.; Schmid, T.; Zenobi, R.; Martin, O. J. F. Mode-Selective Surface-Enhanced Raman Spectroscopy Usingnanofabricated Plasmonic Dipole Antennas. J. Phys. Chem. C 2009, 113, 14672-14675.
40. Reed, J. M.; Wang, H.; Hu, W.; Zou, S. Shape of Fano Resonance Line Spectra Calculated for Silver Nanorods. Opt. Lett. 2011, 36, 4386-4388.

41. Artar, A.; Yanik, A. A.; Altug, H. Directional Double Fano Resonances in Plasmonic Hetero-Oligomers. Nano Lett. 2011, 11, 3694-3700.

42. Yang, Z.-J.; Zhang, Z.-S.; Zhang, W.; Hao, Z.-H.; Wang, Q.-Q. Twinned Fano Interferences Induced by Hybridized Plasmons in Au-Ag Nanorod Heterodimers. App. Phys. Lett 2010, 96, 131113.

43. Yang, Z.-J.; Zhang, Z.-S.; Zhang, L.-H.; Li, Q.-Q.; Hao, Z.-H.;Wang, Q.-Q. Fano Resonances in Dipole-Quadrupole Plasmon Coupling Nanorod Dimers. Opt. Lett. 2011, 36, 1542-1544.

44. Shafiei, F.; Monticone, F.; Le, K.; Liu, X.-X.; Hartsfield, T.; Alù, A.; Li, X. A Subwavelength Plasmonic Metamolecule Exhibiting Magnetic-Based Optical Fano Resonance. Nat. Nanotechnol. 2013, 8, 95-99.

45. Fan, S.; Suh, W.; Joannopoulos, J. D. Temporal CoupledMode Theory for the Fano Resonance in Optical Resonators. J. Opt. Soc. Am. A 2003, 20, 569-572.

46. Ruan, Z.; Fan, S. Temporal Coupled-Mode Theory for Fano Resonance in Light Scattering by a Single Obstacle. J. Phys. Chem. C 2010, 114, 7324-7329.

47. Giannini, V.; Francescato, Y.; Amrania, H.; Phillips, C. C.; Maier, S. A. Fano Resonances in Nanoscale Plasmonic Systems: A Parameter-Free Modeling Approach. Nano Lett. 2011, 11, 2835-2840.

48. Gallinet, B.; Martin, O. J. F. Ab Initio Theory of Fano Resonances in Plasmonic Nanostructures and Metamaterials. Phys. Rev. B 2011, 83, 235427.

49. Francescato, Y.; Giannini, V.; Maier, S. A. Plasmonic Systems Unveiled by Fano Resonances. ACS Nano 2012, 6, 18301838.

50. Joe, Y. S.; Satanin, A. M.; Kim, C. S. Classical Analogy of Fano Resonances. Phys. Scr. 2006, 74, 259-266.

51. Rahmani, M.; Lukiyanchuk, B.; Ng, B.; Tavakkoli K. G., A.; Liew, Y. F.; Homg, M. H. Generation of Pronounced Fano Resonances and Tuning of Subwavelength Spatial Light Distribution in Plasmonic Pentamers. Opt. Express 2011, 19, 4949-4956.

52. Alzar, C. L. G.; Martinez, M. A. G.; Nussenzweig, P. Classical Analog of Electromagnetically Induced Transparency. Am. J. Phys. 2001, 70, 4.

53. Klimov, V. Nanoplasmonics; PanStanford Publishing: Singapore, 2012.

54. Lassiter, J. B.; Sobhani, H.; Fan, J. A.; Kundu, J.; Capasso, F.; Nordlander, P.; Halas, N. J. Fano Resonances in Plasmonic Nanoclusters: Geometrical and Chemical Tunability. Nano Lett. 2010, 10, 3184-3189.

55. Christ, A.; Ekinci, Y.; Solak, H. H.; Gippius, N. A.; Tikhodeev, S. G.; Martin, O. J. F. Controlling the Fano Interference in a Plasmonic Lattice. Phys. Rev. B 2007, 76, 201405.

56. Friedrich, H.; Wintgen, D. Physical Realization of Bound States in the Continuum. Phys. Rev. A 1985, 31, 3964-3966.

57. Friedrich, $\mathrm{H}_{\text {; }}$ Wintgen, D. Interfering Resonances and Bound States in the Continuum. Phys. Rev. A 1985, 32, 3231-3242.

58. Kern, A. M.; Martin, O. J. F. Surface Integral Formulation for 3d Simulations of Plasmonic and High Permittivity Nanostructures. J. Opt. Soc. Am. A 2009, 26, 732-740.

59. Gallinet, B.; Martin, O. J. F. Scattering on Plasmonic Nanostructures Arrays Modeled with a Surface Integral Formulation. Photonics Nanostruct.: Fundam. Appl. 2010, 8, 278284

60. Johnson, P. B.; Christy, R. W. Optical Constants of the Noble Metals. Phys. Rev. B 1972, 6, 4370-4379.

61. Novotny, L. Strong Coupling, Energy Splitting, and Level Crossings: A Classical Perspective. Am. J. Phys. 2010, 78, 1199-1202.

62. Kern, A. M.; Martin, O. J. F. Pitfalls in the Determination of Optical Cross Sections from Surface Integral Equation Simulations. IEEE Trans. Antennas Propag. 2010, 58, 21582161.

63. Zhang, S.; Ye, Z.; Yuan, W.; Park, Y.; Bartal, G.; Mrejen, M.; Yin, X.; Zhang, X. Anti-Hermitian Plasmon Coupling of an 
Array of Gold Thin-Film Antennas for Controlling Light at the Nanoscale. Phys. Rev. Lett. 2012, 109, 193902.

64. Kats, M. A.; Yu, N.; Genevet, P.; Gaburro, Z.; Capasso, F. Effect of Radiation Damping on the Spectral Response of Plasmonic Components. Opt. Express 2011, 19, 2174821753.

65. Gallinet, B.; Martin, O. J. F. Relation between Near-Field and Far-Field Properties of Plasmonic Fano Resonances. Opt. Express 2011, 9, 22167-22175.

66. Zhang, W.; Gallinet, B.; Martin, O. J. F. Symmetry and Selection Rules for Localized Surface Plasmon Resonances in Nanostructures. Phys. Rev. B 2010, 81, 233407.

67. Lassiter, J. B.; Sobhani, H.; Knight, M. W.; Mielczarek, W. S.; Nordlander, P.; Halas, N. J. Designing and Deconstructing the Fano Lineshape in Plasmonic Nanoclusters. Nano Lett. 2012, 12, 1058-1062.

68. Luk'yanchuk, B.; Zheludev, N. I.; Maier, S. A.; Halas, N. J.; Nordlander, P.; Giessen, H.; Chong, C. T. The Fano Resonance in Plasmonic Nanostructures and Metamaterials. Nat. Mater. 2010, 9, 707-715. 


\section{Supporting information}

\section{Mechanisms of Fano Resonances in Coupled \\ Plasmonic Systems}

Andrea Lovera, $\dagger$ Benjamin Gallinet, $\dagger$ Peter Nordlander, $\neq$ Olivier J.F. Martin * $\dagger$

$\dagger$ Nanophotonics and Metrology Laboratory, Swiss Federal Institute of Technology (EPFL), CH-

1015 Lausanne

$\$$ Department of Physics and Astronomy, Rice University, MS 61, Houston, Texas 77005,

United States

*Address correspondence to: olivier.martin@epfl.ch 

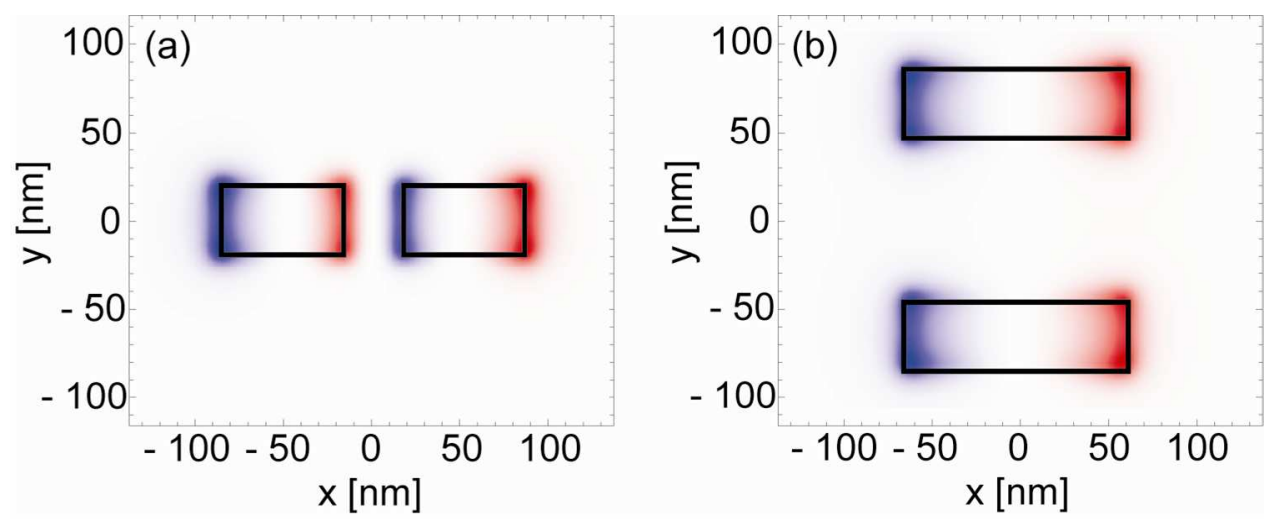

Figure S1. Near-field maps of isolated (a) antenna with $\mathrm{d}=25 \mathrm{~nm}$ and (b) nanorods with $\mathrm{L}=120 \mathrm{~nm}$ and center to center distance of $100 \mathrm{~nm}$ computed at $\omega=1.18 \mathrm{eV}$. Width and thickness for both geometries are fixed to $40 \mathrm{~nm}$. The field is sampled $5 \mathrm{~nm}$ above the structures and the real part of the normal component is plotted. This provides a simple way of analyzing the charge distribution inside the plasmonic structures.

(a)
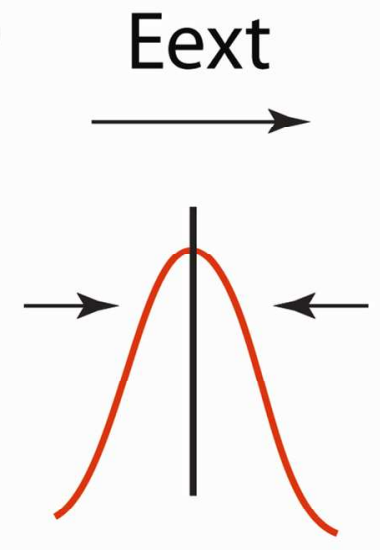

(b) Eext
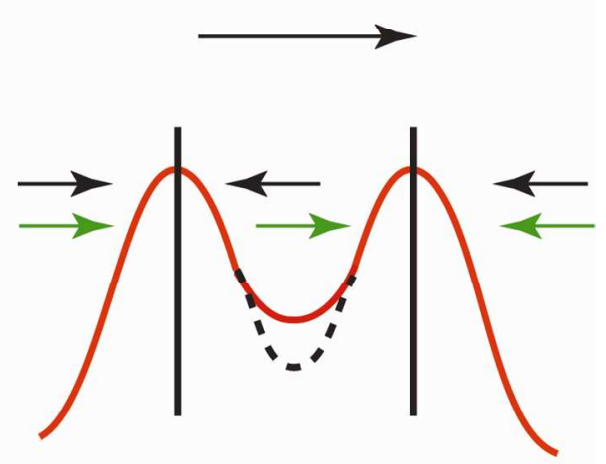

Figure S2. (a) Schematics of the instantaneous dipole orientation on the two sides of a dipole resonance as response of an external field. (b) Schematics of the interaction of two dipole resonances along with their instantaneous field (black and green arrows). In the region where the dipole oscillate out-of-phase we observe destructive interference and higher modulation depth (dashed line). 


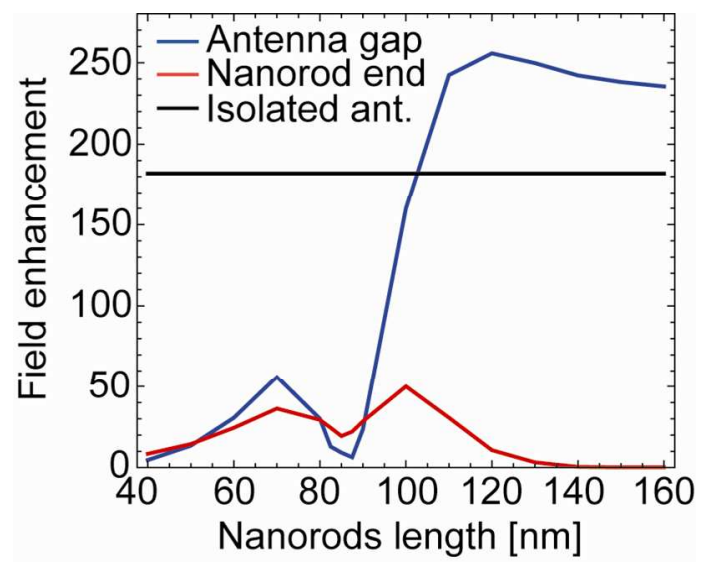

Figure S3. Electric field simulations at AD frequency in the center of the antenna gap (blue) and $5 \mathrm{~nm}$ far from one nanorod end (red), compared with the field computed in the center of the gap for a single antenna without nanorods around.
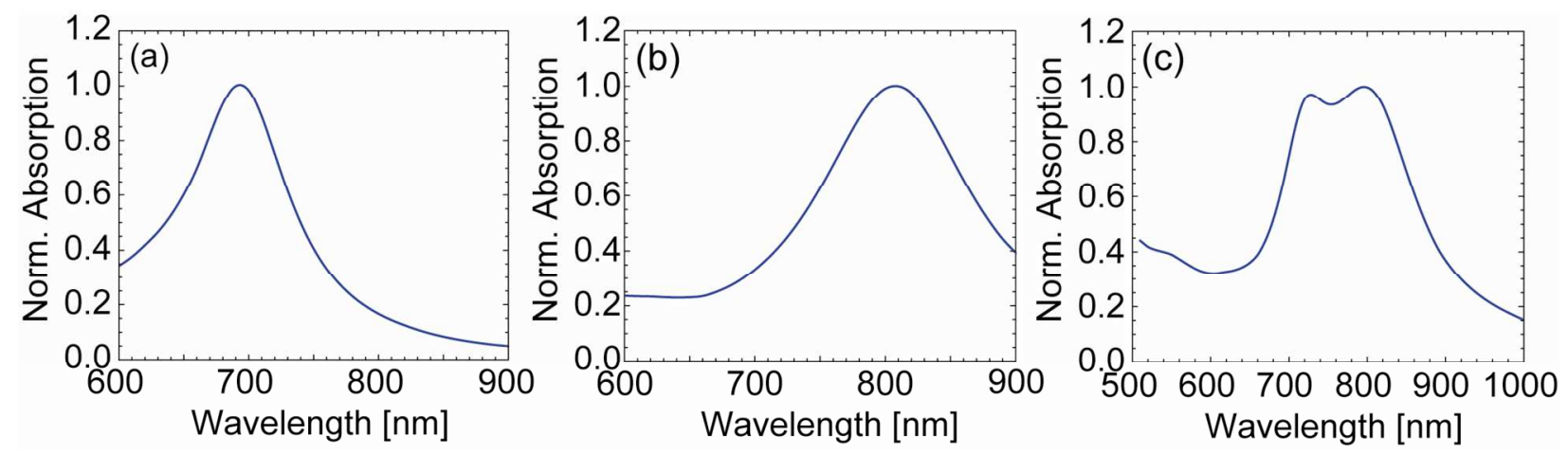

Figure S4. Absorption cross-section for uncoupled (a) nanoantenna with $d=25 \mathrm{~nm}$ and (b) nanorods with $\mathrm{L}=$ $120 \mathrm{~nm}$ and center-center distance of $100 \mathrm{~nm}$. Width and thickness for both geometries are fixed to $40 \mathrm{~nm}$. (c) Absorption for the structure composed by the coupled structure.
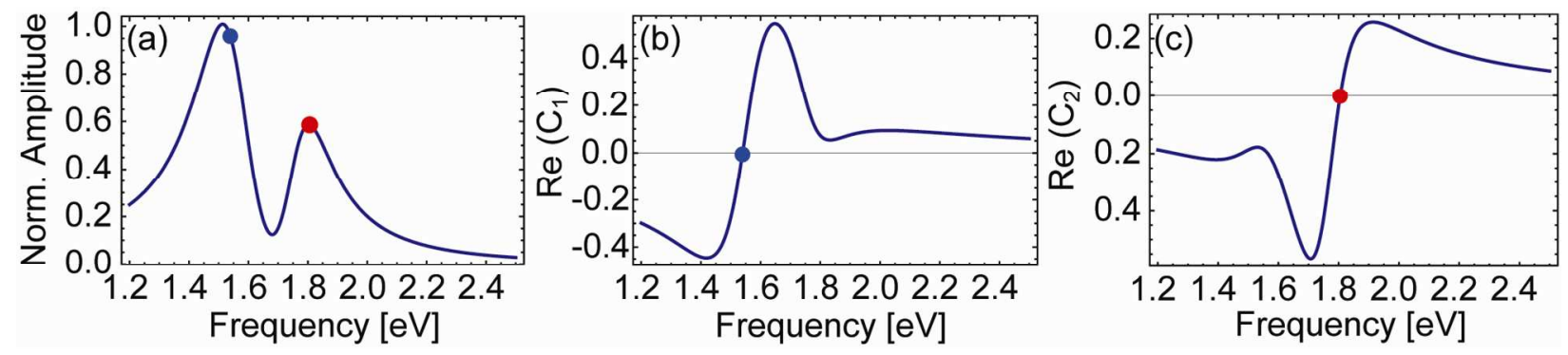

Figure S5. (a) Reconstruction from the oscillator model of the scattering cross-section. (b) Real part of $\mathrm{C}_{1}$ and (c) real part of $\mathrm{C}_{2}$. 

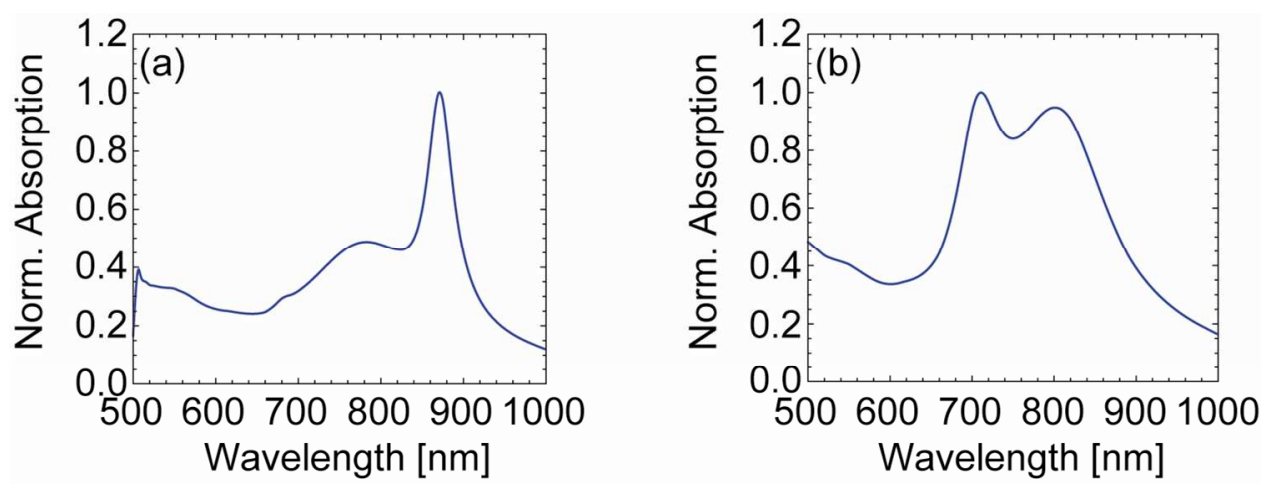

Figure S6. Absorption cross-section for a structure with $\mathrm{L}=120 \mathrm{~nm}$ and antenna $\mathrm{d}=$ (a) $5 \mathrm{~nm}$ and (b) $30 \mathrm{~nm}$. The narrow and wide resonances are inverted in wavelength when comparing both cases. 